\title{
Diagnóstico e proposta de gerenciamento de resíduos sólidos urbanos para o município de Floresta - PE, Brasil
}

Diagnosis and proposal for urban solid waste management for the municipality of Floresta - PE, Brazil

Diagnóstico y propuesta de manejo de residuos sólidos urbanos para el municipio de Floresta - PE,

Jamile Maria Pereira Bastos Lira de Vasconcelos ORCID: https://orcid.org/0000-0002-0898-0643 Secretaria de Educação e Esportes de Pernambuco, Brasil E-mail: jamilebastoslira@gmail.com

Dennis Bezerra Correia ORCID: https://orcid.org/0000-0002-7782-4767 Universidade Regional do Cariri, Brasil E-mail: denniscorreia40@gmail.com

Cícero Jorge Verçosa

ORCID: https://orcid.org/0000-0002-3284-6719 Secretaria de Educação e Esportes de Pernambuco, Brasil E-mail: cjvercosa@hotmail.com

Maria Eliana Vieira Figueroa

ORCID: https://orcid.org/0000-0002-0049-4456 Secretaria de Educação e Esportes de Pernambuco, Brasil E-mail: elianavfigueroa1@gmail.com

Elizângela Beneval Bento

ORCID: https://orcid.org/0000-0002-7516-9711 Universidade Regional do Cariri, Brasil E-mail: elizangelaeliz@yahoo.com.br

Luiz Neldecílio Alves Vitor

ORCID: https://orcid.org/0000-0003-4085-4214 Universidade Federal do Cariri, Brasil

E-mail: professorluizneldecilio@gmail.com João Eudes Lemos de Barros

ORCID: https://orcid.org/0000-0003-1829-795X Universidade Regional do Cariri, Brasil E-mail: joao.eudeslemos@urca.br

João Paulo Camilo de Oliveira ORCID: https://orcid.org/0000-0003-0286-1149 Universidade Regional do Cariri, Brasil E-mail: camilodeoliveirajoaopaulo35@gmail.com

José Aglailson Oliveira da Anunciação ORCID: https://orcid.org/0000-0003-1751-2953

Universidade Regional do Cariri, Brasil E-mail: aglailsonoliveira@gmail.com Luciano Temoteo dos Santos ORCID: https://orcid.org/0000-0002-9215-6832 Universidade Federal do Cariri, Brasil

E-mail: luciano.temoteosantos@gmail.com

Alice Fernandes Gusmão

ORCID: https://orcid.org/0000-0003-2778-248X Universidade Regional do Cariri, Brasil E-mail: alicefg55@gmail.com

\section{Resumo}

$\mathrm{O}$ aumento populacional nos centros urbanos representa um grande desafio, pois à medida que a população aumenta mais áreas serão desmatadas para a construção de moradias, mais materiais e bens de consumos são gerados para atender às necessidades da população. As atividades em que os homens realizam, resultam em resíduos que na grande maioria são liberados inadequadamente, comprometendo o meio ambiente. A liberação desses resíduos e o não tratamento deles contribuem para a degradação da biosfera, impossibilitando a qualidade de vida no planeta e possibilitando a 
proliferação de doenças para a população. O mau gerenciamento dos resíduos sólidos urbanos tem obrigado os municípios brasileiros a encontrarem alternativas para esse gerenciamento e disposição final desses materiais. Essa pesquisa expõe a situação atual dos RSU do município de Floresta - PE. Os resultados obtidos evidenciam que o tratamento e disposição final dos resíduos sólidos do município são de forma inadequada, gerando impactos ambientais e sociais para a população. Os dados obtidos nessa pesquisa servirão para subsidiar a construção de um plano de gerenciamento de resíduos sólidos e educação ambiental no município com ações coletivas a serem desenvolvidas com a população.

Palavras-chave: Resíduos sólidos; Gerenciamento; Lixo urbano.

\begin{abstract}
The population increase in urban centers represents a great challenge, as the population increases, more areas will be deforested for housing construction, more materials and consumer goods are generated to meet the population's needs. The activities that men carry out result in residues that are mostly inappropriately released, compromising the environment. The release of these residues and the non-treatment of them contribute to the degradation of the biosphere, impairing the quality of life on the planet and enabling the proliferation of diseases for the population. The poor management of urban solid waste has forced Brazilian municipalities to find alternatives for this management and final disposal of these materials. This research exposes the current situation of MSW in the municipality of Floresta - PE. The results obtained show that the treatment and final disposal of solid waste in the city is inadequate, generating environmental and social impacts for the population. The data obtained in this research will serve to support the construction of a solid waste management plan and environmental education in the municipality with collective actions to be developed with the population.
\end{abstract}

Keywords: Solid waste; Management; Urban garbage.

\title{
Resumen
}

El aumento de población en los centros urbanos representa un gran desafío, ya que a medida que aumenta la población se deforestarán más áreas para la construcción de viviendas, se generarán más materiales y bienes de consumo para satisfacer las necesidades de la población. Las actividades que realizan los hombres dan como resultado residuos que en su mayoría se liberan de manera inapropiada, comprometiendo el medio ambiente. La liberación de estos residuos y el no tratamiento de los mismos contribuyen a la degradación de la biosfera, perjudicando la calidad de vida en el planeta y posibilitando la proliferación de enfermedades para la población. La mala gestión de los residuos sólidos urbanos ha obligado a los municipios brasileños a buscar alternativas para esta gestión y disposición final de estos materiales. Esta investigación expone la situación actual de los RSU en el municipio de Floresta - PE. Los resultados obtenidos muestran que el tratamiento y disposición final de los residuos sólidos en la ciudad es inadecuado, generando impactos ambientales y sociales para la población. Los datos obtenidos en esta investigación servirán para apoyar la construcción de un plan de manejo de residuos sólidos y educación ambiental en el municipio con acciones colectivas a desarrollar con la población.

Palabras clave: Residuos sólidos; Gestión; Basura urbana.

\section{Introdução}

De acordo com Odum (1988), um ecossistema é formado pelo conjunto de fatores bióticos e um conjunto de fatores abióticos tendo uma relação direta entre os organismos vivos com os fatores físicos do ambiente.

O ecossistema urbano compreende as cidades e os grandes centros urbanos e é onde as alterações são mais significativas e se caracterizam por: alta densidade demográfica. O aumento populacional nos centros urbanos representa um grande desafio, pois à medida que a população aumenta mais áreas serão desmatadas para a construção de moradias, mais materiais e bens de consumos são gerados e a disponibilidade de água tem que ser maior para atender às necessidades da população (Melo et al, 2019).

As atividades em que os homens realizam, resultam em resíduos que na grande maioria são liberados inadequadamente, comprometendo o meio ambiente (Farias, 2020). O crescendo populacional traz uma perspectiva de aumento de resíduos, pois, a quantidade de alimentos e bens de consumo tem que ser maior. A liberação desses resíduos e o não tratamento deles contribuem para a degradação da biosfera, impossibilitando a qualidade de vida no planeta (Pozzetti \& Caldas, 2019). 
A maioria das cidades é comum observamos hábitos de disposição final inadequados de lixo. Materiais sem utilidade se amontoam desordenadamente, muitas vezes em locais indevidos como lotes baldios, margens de estradas, fundos de vale e margens de lagos e rios (Freitas et al, 2020).

O gerenciamento do resíduo sólido é um desafio para a gestão pública, a problemática do lixo com a coleta realizada pela administração municipal ou empresas particulares, dando o despejo final desses resíduos que surgem os problemas ambientais, principalmente pelo pequeno número de cidades que possuem aterros adequados. (Tagliber, 2002).

O lixo urbano na maioria das vezes é o grande responsável por impactos ambientais principalmente em áreas urbanas (Conceição et al, 2020). Sendo assim, o objetivo foi identificar as como se dá o gerenciamento dos resíduos sólidos e os impactos gerados pelo lixo no município de Floresta- PE. Com isso, o trabalho tem como justificativa apresentar possíveis soluções para o gerenciamento dos resíduos a fim de propor alternativas economicamente viáveis, que possam servir de soluções imediatas e permanentes para a problemática dos RSU no município.

A preocupação com a destinação dos resíduos gerados pelas atividades antrópicas é uma questão mundial (Komatsu \& Sousa, 2019). Para isso, torna-se necessário o controle e gerenciamento desses resíduos através de mecanismos que tornem a gestão de resíduos sólidos eficiente. No Brasil um passo importante foi dado com a Política Nacional dos Resíduos Sólidos (PNRS) instituída pela Lei 12305/10.

De acordo com a PNRS, resíduos sólidos seria o material que após o uso para o qual foi concebido, em seu descarte ainda possui aproveitamento. Diferentemente de rejeito que é considerado como o resíduo que, depois de esgotadas todas as possibilidades de valorização, devem ser dispostos em aterros.

Segundo a norma brasileira NBR 10004, de 1987 - Resíduos sólidos - classificação, resíduos sólidos são:

"aqueles resíduos nos estados sólido e semissólido, que resultam de atividades da comunidade de origem industrial, doméstica, hospitalar, comercial, agrícola, de serviços e de varrição. Ficam incluídos nesta definição os lodos provenientes de sistemas de tratamento de água, aqueles gerados em equipamentos e instalações de controle de poluição, bem como determinados líquidos cujas particularidades tornem inviável o seu lançamento na rede pública de esgotos ou corpos de água, ou exijam para isso soluções técnicas e economicamente inviáveis em face a melhor tecnologia disponível".

Segundo Fonseca (2001), tanto a origem quanto a composição do lixo, são elementos fundamentais para classificá-lo. Quanto à origem esses resíduos podem ser: residencial, comercial, industrial, dos serviços de saúde, especial (restos de podação, animais mortos etc.), público e outros (resíduos como lodos de estação de tratamento de águas e esgotos).

Outras definições importantes também no gerenciamento dos resíduos são os termos destinação e disposição final (Veras \& Faria, 2019). Destinação se refere ao destino que é tomado após o descarte que pode ser a reciclagem, reutilização, compostagem e a recuperação. Já disposição final compreende a organização e disposição dos rejeitos em aterros.

Com o aumento populacional, um dos problemas mais sérios enfrentados pela humanidade é o lixo urbano (Fonseca, 2001). Estima-se que a população mundial hoje de mais de 6 bilhões de habitantes, esteja gerando mais de 30 milhões de toneladas de lixo por ano (D’ Almeida, 2000). Dessa forma, consequentemente, aumentam as áreas de disposição final dos resíduos, gerando assim problemas pra o ambiente como:

$\checkmark$ Danos causados pelo chorume (liquido decorrente da decomposição do lixo);

$\checkmark$ Odor;

$\checkmark$ Fogo e fumaça decorrentes da combustão dos materiais do lixo;

$\checkmark \quad$ Vetores e doenças (roedores, insetos, bactérias vírus).

A Tabela 1 abaixo mostra algumas enfermidades decorrentes da disposição inadequada dos lixos nos centros urbanos. 
Tabela 1: Enfermidades decorrentes da disposição inadequada dos lixos.

\begin{tabular}{l|l}
\hline \multicolumn{1}{c|}{ Vetores } & \multicolumn{1}{c}{ Enfermidades } \\
\hline Ratos e Pulgas & Leptospirose, Peste Bubônica, Tifo Murino \\
\hline Moscas & Febre Tifóide, Cólera, Amebíase, Disenteria, Giardíase, Ascaridíase \\
\hline Mosquito & $\begin{array}{l}\text { Malária, Febre Amarela, Dengue, Leishmaniose, Febre Tifóide, } \\
\text { Cólera }\end{array}$ \\
\hline Barata & Giardíase \\
\hline Gado e Porco & Teníase, Cistecercose \\
\hline Cão e Gato & Toxoplasmose \\
\hline
\end{tabular}

Fonte: Adaptado de Lima (2003).

Segundo dados da Pesquisa Nacional de Saneamento Básico (PNSB) realizada pelo Instituto Brasileiro de Geografia e Estatística (IBGE) em 2002, a população Brasileira produz diariamente cerca de 126 mil toneladas de resíduos sólidos, onde 63,6\% dos municípios brasileiros depositam seus resíduos sólidos em "lixões", 13,8\% utilizam aterros sanitários e 18,4\% dispõem seus resíduos em aterros controlados. Verificou-se ainda na pesquisa que a destinação mais utilizada ainda é o depósito de resíduos sólidos a céu aberto na maioria dos municípios com população inferior a 10.000 habitantes, considerados de pequeno porte, correspondendo a cerca de $48 \%$ dos municípios brasileiros. Nesses municípios, 63,6\% dos resíduos sólidos coletados são depositados em lixões, enquanto $16,3 \%$ são encaminhados para aterros controlados.

Nos lixões os resíduos são colocados desordenadamente, sem cobertura no solo que o proteja do chorume produzido e dessa forma contribuindo para a poluição do solo, de aquíferos, proliferação de vetores e doenças (Moura et al, 2020). Além das questões ambientais, existem os efeitos sociais negativos para a população, uma vez que muitos familiares se instalam nas regiões dos lixões para coletar os resíduos e fazerem uso para alimentação e consumo.

A predominância dos lixões a céu aberto deve-se ao fato de ser um método de baixo custo ou mesmo pela falta de estrutura organizacional das instituições públicas envolvidas com a questão nos municípios, o que acaba refletindo na inexistência ou inadequação de planos de gerenciamento de resíduos sólidos (Mendonça et al, 2019). Para reverter essa realidade nos municípios brasileiros, se fazem necessárias ações, projetos e tecnologias para que a destinação final dos resíduos sólidos seja de forma sustentável, que atenda tanto as condições de cada município quanto às questões ambientais, políticas, financeiras, sociais e que seja integrada com um Plano de Gerenciamento (Santos \& Marchesini, 2018).

De acordo com D’Almeida e Vilhena (2000), o aterro controlado é uma técnica de disposição de resíduos sólidos municipais no solo que busca minimizar os impactos ambientais da disposição a céu aberto. Nesse método, é utilizado métodos da engenharia para confinação dos resíduos, cobrindo-os com material inerte. Não há impermeabilização na base (o que compromete o solo e as águas subterrâneas) e nem sistema de tratamento.

Aterro Sanitário é "uma técnica de disposição de resíduos sólidos urbanos no solo sem causar danos à saúde pública e à sua segurança, minimizando os impactos ambientais, método este que utiliza princípios de engenharia para confinar os resíduos sólidos à menor área possível e reduzi-los ao menor volume permissível, cobrindo-os com uma camada de terra na conclusão de cada jornada de trabalho, ou a intervalos menores, se for necessário" (ABNT NBR 8.419,1984).

O gerenciamento dos resíduos implica um conjunto de ações que visem minimizar os efeitos dos resíduos no ambiente com métodos de visem manter a qualidade ambiental e a melhor qualidade de vida para a população (Pozzetti \& Caldas, 2019). Segundo Guadagnin et al (2018), o gerenciamento do lixo municipal é um conjunto articulado de ações normativas, operacionais, financeiras e de planejamento que uma administração municipal desenvolve (com base em critérios sanitários, ambientais e econômicos), para coletar, segregar, tratar e dispor o lixo de sua cidade. 
A Constituição Federal, promulgada em 1988, estabelece em seu artigo 23, inciso VI, que "compete à União, aos Estados, ao Distrito Federal e aos Municípios proteger o meio ambiente e combater a poluição em qualquer das suas formas”. No artigo 24, estabelece a competência da União, dos Estados e do Distrito Federal em legislar concorrentemente sobre “(...) proteção do meio ambiente e controle da poluição" (inciso VI) e, no artigo 30, incisos I e II, estabelece que cabe ainda ao poder público municipal "legislar sobre os assuntos de interesse local e suplementar a legislação federal e a estadual no que couber" (IPT/Cempre, 2000).

Sendo assim, a prestação do serviço público de limpeza urbana pelo município é muito importante e faz necessária eficiência e qualidade nos serviços para que se evitem danos ao meio ambiente e à saúde da população. Para isso, cada comunidade deve adequar seus serviços para atende à população e ao meio ambiente com responsabilidade e de forma adequada. A Tabela 2 abaixo mostra a qual órgão compete à responsabilidade pelo destino final dos resíduos gerados.

Tabela 2: Responsabilidade pelo gerenciamento de cada tipo de resíduo.

\begin{tabular}{l|l}
\hline \multicolumn{1}{c|}{ Tipos de Lixo } & \multicolumn{1}{c}{ Responsável } \\
\hline Domiciliar & Prefeitura* \\
\hline Comercial & Prefeitura* \\
\hline De serviço & Prefeitura* \\
\hline Industrial & Gerador (indústria) \\
\hline Serviços de Saúde & Gerador (hospitais, etc) \\
\hline Agrícolas & Gerador (agricultor) \\
\hline Entulho & Gerador \\
\hline Radioativo & CNEN \\
\hline
\end{tabular}

(*) a Prefeitura é corresponsável por pequenas quantidades e de acordo com a legislação municipal específica. Fonte: Adaptado de JARDIM et al. (1995).

Algumas legislações foram criadas afim de normatizar o gerenciamento dos resíduos sólidos: (Schalch \& Wendland, 2003).

-Portaria n 53, de 1 de março de 1979 - estabelece normas aos projetos específicos de tratamento e disposição de resíduos sólidos, bem como a fiscalização de sua implantação, operação e manutenção.

-Resolução Conama n ${ }^{\circ} 275$, de 25 de abril de 2001 - dispõe sobre coleta seletiva, impacto ambiental, reciclagem, tipos de resíduos, recursos naturais, energia, água, beneficiamento, transporte, tratamento, aterro sanitário, lixões, campanhas, educação ambiental, códigos de cores, órgãos, iniciativa privada, cooperativas, organizações não-governamentais, entidades, padronização, entre outros.

No estado de Pernambuco existe o Plano Estadual de Resíduos Sólidos (PERS) que é pautado em conjunto de diretrizes e estratégias para subsidiar a gestão dos resíduos sólidos do estado. A Política Estadual de Resíduos Sólidos foi instituída pela Lei $N^{\circ}$ 14.236, de 13 de dezembro de 2010. A missão da PERS é enfrentar o passivo ambiental do estado decorrente da coleta e da destinação final dos resíduos sólidos.

Alguns dos objetivos da PERS do estado de Pernambuco são: proteger o meio ambiente, garantir o uso racional dos recursos naturais e estimular a recuperação de áreas degradadas; implementar a gestão integrada de resíduos sólidos; promover ações de educação ambiental, especialmente quanto ao descarte adequado dos resíduos por parte da coletividade; promover ações voltadas à inclusão social de catadores de materiais recicláveis; disseminar informações relacionadas à gestão dos resíduos sólidos; incentivar a pesquisa, o desenvolvimento, a adoção e a divulgação de novas tecnologias de reciclagem e compostagem, tratamento, destinação e disposição final de resíduos sólidos, inclusive de prevenção à poluição (PERS, 2012). 
O PERS de Pernambuco oferece dados e estratégias de ações para o gerenciamento dos resíduos sólidos nos municípios. Com relação a RD do Sertão de Itaparica, é apresentada uma tabela de Composição Gravimétrica dos resíduos sólidos (Tabela 3). De acordo com os dados apresentados, é possível perceber o como se dá a porcentagem dos tipos de resíduos.

Tabela 3: Composição Gravimétrica (2010) (\%).

\begin{tabular}{c|c|c|c|c|c|c|c}
\hline Município & Matéria Orgânica & Vidro & Metal & Papel & Plástico & Reciclável & Rejeitos \\
\hline Belém do São Francisco & & & & & & \\
& 62,00 & 2,90 & 4,00 & 7,60 & 10,40 & 24,90 & 13,10 \\
\hline Carnaubeira da Penha & 43,00 & 0,00 & 3,00 & 19,00 & 8,00 & 30,00 & 27,00 \\
\hline Floresta & 52,30 & 3,00 & 2,60 & 11,60 & 13,90 & 31,10 & 16,90 \\
\hline Itacuruba & 62,00 & 3,00 & 3,80 & 9,90 & 8,40 & 25,10 & 12,90 \\
\hline Jatobá & 54,00 & 3,10 & 3,80 & 10,30 & 9,00 & 26,20 & 19,80 \\
\hline Petrolândia & 61,00 & 3,40 & 4,50 & 9,00 & 9,60 & 26,50 & 12,50 \\
\hline
\end{tabular}

Fonte: Adaptado de PERS.

Ao se observar o volume de resíduos produzidos, percebe-se claramente a necessidade de implementação de estratégias de gerenciamento de resíduos sólidos na região.

O PERS dispõe ainda de tabelas que demonstram as projeções de produção de resíduos sólidos por município. Para a cidade de Floresta, a Tabela 3 demonstra o quantitativo de resíduos sólidos produzidos em toneladas por ano (t/ano).

Tabela 4: Projeções de produção de Resíduos Sólidos (t/ano) para a cidade de Floresta-PE.

\begin{tabular}{|c|c|}
\hline Ano & Projeções de Produção de Resíduos Sólidos (t/ano) \\
\hline 2016 & $8.735,36$ \\
\hline 2020 & $9.051,00$ \\
\hline 2024 & $9.307,04$ \\
\hline 2028 & $9.539,82$ \\
\hline 2032 & $9.749,00$ \\
\hline
\end{tabular}

Fonte: Adaptado de PERS.

De acordo com a Tabela 4, as projeções para o futuro são de aumento na quantidade de resíduos produzidos pela população da cidade de Floresta. Medidas de gerenciamento e de educação ambiental são essenciais para evitar danos ambientais e doenças ocasionadas pelo lixo.

\section{Metodologia}

\section{Tipo de pesquisa}

A princípio foram realizadas pesquisas bibliográficas sobre o objeto de estudo, através de consultas em literaturas específicas (pesquisa em artigos e livros), consultas em banco de dados (IBGE e PERS) e a profissionais responsáveis pela limpeza pública da prefeitura Municipal de Floresta. Em seguida foram realizadas visitas técnicas ao local de disposição final dos resíduos sólidos urbanos do município.

A pesquisa procurou-se a observar a situação atual do gerenciamento dos serviços de limpeza pública e destinação dos Resíduos Sólidos Urbanos (RSU), a fim de avaliar os danos ao meio ambiente decorrentes da disposição final dos resíduos e 
propor alternativas para mitigar os problemas ambientais e sociais.

Foram feitas visitas e entrevistas a profissionais responsáveis pela limpeza pública do município, no entanto não foi seguida na pesquisa uma metodologia padrão para a entrevista, porém foram seguidas as seguintes etapas:

$\checkmark$ A elaboração do formulário para a pesquisa foi feita primeiramente com perguntas simples sobre gerenciamento dos resíduos sólidos, passando gradativamente a perguntas mais complexas, o formulário foi composto por 15 perguntas objetivas;

$\checkmark$ Foi investigado como ocorre o procedimento de coleta, limpeza, transporte e destinação final dos resíduos;

$\checkmark$ Foram investigados também se existem no município projetos que incentivem a coleta seletiva, palestras de conscientização ambiental com a população e programas de educação ambiental nos espaços públicos (escolas, feira livre);

$\checkmark$ Houve a visita ao espaço onde ocorre a destinação "final" dos resíduos sólidos, o lixão, entrevistando catadores e moradores dos arredores.

As informações obtidas foram analisadas e apresentadas como resultado para discussões acadêmicas.

\section{Resultados e Discussão}

Floresta é uma cidade do sertão pernambucano, com 32.152 habitantes. A geração de Resíduos Sólidos Urbanos - RSU é bastante razoável e compatível com o padrão de vida de seus habitantes. Maior parte dos resíduos gerados tem como fonte geradora as residências. Estes resíduos possuem grande potencial de reaproveitamento: as latas de alumínio, os papeis e as garrafas PET entre outros. Com isso, pode haver projetos que viabilizem e estimulem a coleta seletiva e a reciclagem, visto que a cidade possui boa estrutura para o desenvolvimento de projetos sociais voltados para a melhoria da qualidade de vida da população.

No âmbito ambiental, a gestão municipal possui alguns desafios para o gerenciamento dos seus resíduos. Algumas delas é a conscientização da população sobre deposição final dos resíduos e o fator econômico que impede a cidade ter um local adequado para disposição final dos resíduos (aterro sanitário). Muitos habitantes ainda não demonstram interesse em colaborar com a limpeza pública e despejam seus resíduos em qualquer local. No entanto, a gestão municipal mostra-se interessada em adequar seus serviços para realizar modificações administrativas, adequando sua maneira de manejar os RSU, em conformidade com as leis ambientais e estimular a população a ter uma Educação Ambiental.

$\mathrm{Na}$ análise e no diagnostico realizado in loco, foi possível perceber os efeitos ambientais relacionados à gestão dos resíduos sólidos, onde se observou a manipulação inadequada desses resíduos. Além desse fato, foi possível perceber:

- falta de locais adequados para a deposição final dos resíduos (aterro sanitário);

- os resíduos coletados pelo carro apropriados para a coleta, são levados ao lixão onde são dispostos de forma aleatória;

- ausência de lixeiras que incentive a coleta seletiva nos pontos públicos da cidade;

- ausência de projetos que incentivem a reciclagem;

- ausência de documentação que demonstrasse o cumprimento de determinadas exigências legais, como um plano de gerenciamento de resíduos sólidos para o município;

- que os RSU estão sendo dispostos sem nenhum cuidado ou técnica apropriada, caracterizando a área como lixão do município (Imagens 2 e 3); 
Imagem 1: Disposição dos RSU.

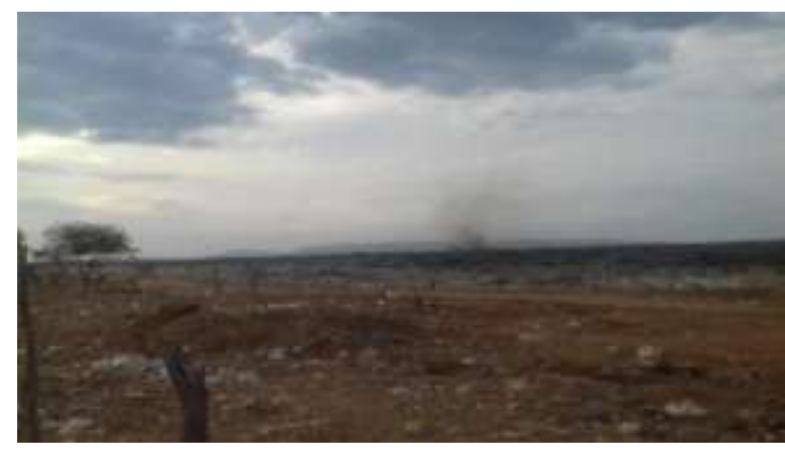

Imagem 2: Disposição dos RSU.



Fonte: Autores.

os resíduos colocados no lixão não são concentrados em um único ponto, são espalhados, o que propicia que eles sejam levados pelo vento para outros locais;

$\checkmark$ família residindo no lixão e se beneficiando nos resíduos do local (Imagem 4). Em entrevista realizad, pais de família que moram no local relataram que dependem totalmente dos resíduos para a alimentação e que as crianças que residem no local, apresentam ou já apresentaram enfermidades decorrentes do lixo;

Imagem 3: Moradias perto da disposição dos RSU.



Fonte: Autores.

a fumaça presente no local é decorrente da combustão dos materiais existentes no lixo;

$\checkmark$ foi observado que apenas os resíduos sólidos dos serviços de saúde estão recebendo adequado tratamento, onde os mesmos são incinerados em outra cidade;

$\checkmark$ que a cidade está crescendo para os locais da periferia e que existem loteamentos próximos à área do lixão. Com o tempo, a existência do lixão poderá levar vetores de doenças para as famílias;

$\checkmark$ em entrevista com o responsável pela limpeza pública, que a prefeitura busca a qualidade dos serviços. Segundo o responsável, no começo do ano de 2016 houve um surto de doenças transmitidas pelo inseto Aedes aegypti e a gestão municipal intensificou os trabalhos de limpeza pública e incentivando a população para evitar o descarte do lixo de 
qualquer modo e em qualquer local;

$\checkmark$ embora área o lixão seja cercado, isso não impede de o lixo sair do espaço e se dispersando para outros locais;

$\checkmark$ falta de projetos permanentes de educação ambiental em espaços públicos (escolas, secretarias, praças) que visem despertar na população os cuidados com o meio ambiente.

$\checkmark$ consultando dados do PERS (2012), que o município de Floresta tem uma produção de resíduos sólidos em toneladas por dia ( $\mathrm{t} / \mathrm{dia}$ ) correspondente a 22,70, por mês ( $\mathrm{t} / \mathrm{mês}$ ) correspondente a 8.286,00. A cidade tem um custo total de serviços de limpeza urbana igual a $\mathrm{R} \$ 458.640,00$ por ano.

A Tabela 5 mostra os resultados obtidos com a pesquisa de campo realizada na área de disposição final dos resíduos sólidos do município de Floresta-PE.

Tabela 5: Resultados obtidos in loco.

\begin{tabular}{c|c}
\hline Aspectos Analisados & Situação \\
\hline Proximidade de núcleos habitacionais & Próximo \\
\hline Cercamento de área & Não \\
\hline Portaria / vigilante & Não \\
\hline Controle de recebimento de carga & Inexistente \\
\hline Drenagem do Chorume & Inexistente \\
\hline Sistema de tratamento de chorume & Sim \\
\hline Presença de Urubus, gaivotas etc & Sim \\
\hline Presença de moscas em grande quantidade & Sim \\
\hline Presença de queimadas & .
\end{tabular}

Fonte: Autores.

Realizados a análise das observações in loco, foram elaboradas estratégias de ação para o adequado gerenciamento dos resíduos sólidos do município de Floresta-Pe, para que resulte na melhoria da qualidade de vida da população e para os cuidados e manejo adequados dos recursos da natureza.

Tendo em vista os resultados obtidos, foram elaboradas propostas de estratégias de ação para que a gestão municipal efetue em médio e em longo prazo. As estratégias buscam incentivar a visão ambiental da população e estimular o manejo adequado do meio ambiente, além de criar núcleos que beneficiem a sociedade com atividades de reciclagem. Sendo assim a proposta de gerenciamento de resíduos urbanos de Floresta-Pe, contempla a problemática dos diversos tipos de resíduos gerados, as alternativas de gestão e gerenciamento passíveis de implementação, tendo como propostas de estratégias de ação:

$\checkmark$ Elaborar o Plano Municipal de Resíduos Sólidos;

$\checkmark$ Reestruturar o local de disposição final dos resíduos sólidos (Lixão), com possível construção de um aterro sanitário ou aterro controlado;

$\checkmark$ Capacitar funcionários que realizam a limpeza pública;

$\checkmark$ Elaborar programas de Coleta Seletiva em órgãos públicos, comercial e em residências, com inclusão dos catadores de materiais recicláveis e reutilizáveis;

$\checkmark \quad$ Instalar coletores de lixo que estimulem a coleta seletiva;

$\checkmark$ Criar locais que realizem a compostagem com os materiais orgânicos coletados;

$\checkmark$ Desenvolver projetos de capacitação da população para a reciclagem, com criação de cooperativa que estimulem a geração de emprego e renda a partir dos produtos feitos com materiais recicláveis; 
$\checkmark$ Integrar ações de Educação Ambiental nos diversos órgãos municipais (escolas; espaços públicos; secretarias de saúde, limpeza pública e educação);

$\checkmark$ Criar espaço público educativo que vise a Educação Ambiental, com palestras, atividades e experimentos a serem desenvolvidos com estudantes e demais atores sociais;

$\checkmark \quad$ Estimular as boas práticas no manejo de resíduos e estabelecer indicadores para o apoio ao acompanhamento, controle social e revisão de planos e programas;

$\checkmark$ Ampliar a conscientização quanto à importância da produção e do consumo responsável e a minimização de resíduos, incentivando a cultura da não geração, reutilização e reciclagem;

$\checkmark$ Produzir e organizar materiais para apoiar ações de educação ambiental;

$\checkmark$ Desenvolver ações de educação ambiental na educação formal e não formal, pública e privada, em todos os níveis e modalidades de ensino, com envolvimento e participação da comunidade escolar (gestores, professores, funcionários, alunos e pais), os prestadores de serviços e/ou multiplicadores para implantação do Programa Escola Sustentável;

$\checkmark$ Criar e apoiar espaços voltados à valorização do reuso, inclusive na administração pública, de conserto ou reaproveitamento de equipamentos, móveis e utensílios;

$\checkmark$ Mobilizar a comunidade, os educadores e instituições, visando a participação ativa pela implantação do gerenciamento dos resíduos sólidos, nas ações articuladas e na observância aos princípios da ética ambiental.

Para que essa proposta de gerenciamento de resíduos sólidos resulte na melhoria das questões ligadas a meio ambiente no município, é necessário o envolvimento dos diversos atores sociais e a mudança de comportamento dos indivíduos nos assuntos relacionados a liberação dos resíduos.

\section{Considerações Finais}

Diante do estudo realizado, conclui-se que os resultados revelados na pesquisa apontam que a atual forma de dispor os RSU no município de Floresta-PE está ocorrendo de maneira inadequada e poderá ocasionar sérios prejuízos ao meio ambiente, à economia e à qualidade de vida da população. Nos dados obtidos ficou evidente que não há estratégias relacionadas à adequação e/ou destinação correta dos resíduos sólidos e nem há previsão de se construir um aterro sanitário e também de se adotar a coleta seletiva, distanciando-se assim da exigibilidade legal.

É necessário que a gestão pública do município reconheça que para haver uma mudança nos problemas sociais e ambientais no município, precisa ser tomada uma decisão eficaz de implementar políticas públicas direcionadas ao bem estar da população, pois para ter um município de acordo com os padrões necessário, é importante que haja investimentos em estratégicas e políticas públicas nas grandezas econômicas, sociais e ambientais, para se ter uma sociedade digna para a população em geral.

\section{Referências}

Conceição, M. M., Costa, R., Conceição, J. T. P., Rosini, A. M., \& Dalma, F. B. (2020). Viabilidade econômica da reciclagem dos resíduos urbanos da cidade de Lisboa-PT-uma análise utilizando o aplicativo verdes-pt. Research, Society and Development, 9(5), e46952961-e46952961.

D’Almeida, M. L. O., \& Vilhena, A. (2000). Lixo municipal: manual de gerenciamento integrado. IPT/Cempre, 2.

Fonseca, E. (2001). Iniciação ao estudo dos resíduos sólidos e da limpeza urbana. Editora União, 122.

Freitas, M. C. C., Barreto, C. A., Santos, G. B., \& Silva Freitas, N. M. (2020). Percepção socioambiental dos residentes do Igarapé Santa Cruz no município de Breves (PA): vivências e desafios sobre a captação e uso da água. Revista Brasileira de Educação Ambiental (RevBEA), 15(1), 328-350.

Guadagnin, M. R., Selau, C. C., \& Cadorin, S. B. (2018). Gestão E Gerenciamento De Resíduos Sólidos No Município De Criciúma/SC. Tecnologia e Ambiente, $24,159-180$.

Instituto Brasileiro de Geografia, Estatística. Departamento de População, \& Indicadores Sociais. (2002). Pesquisa nacional de saneamento básico: 2002. IBGE. 
Komatsu, R. K., dos Santos, C. H. P., \& de Sousa, J. C. (2019). Gestão de Resíduos: hábitos de descarte de resíduos derivados da produção agrícola das propriedades em assentamentos rurais/Waste Management: Waste Disposal Habits from Agricultural Production of Properties in Rural Settlements. ID on line Revista De Psicologia, 13(44), 700-722.

Melo, E., Carasek, M., \& Melo, R. (2019). Ecologia Urbana: vegetação arbórea de um município de pequeno porte. Revista Nacional de Gerenciamento de Cidades, 7(48), 56-69.

Mendonça, D. D. S. M., Zang, J. W., \& Da Fonseca-Zang, W. A. (2017). Efeitos e danos ambientais da disposição de resíduos sólidos na área do lixão e aterro controlado no município de Inhumas-GO. Caderno de Geografia, 27(50), 486-499.

Moura, R. S. C., Estevam, S. M., de Queiroz Fernandes, A. C., de Almeida Oliveira, P., Sarmento, R. J. G., de Oliveira Barbosa, W., \& da Silva Araújo, D. (2020). Políticas públicas e gestão de resíduos sólidos urbanos no Município de Rafael Fernandes/RN. Research, Society and Development, 9(9), e470997598e470997598.

NBR (2016). 8.419 1984. Aterro Sanitário.

NBR, A. (2004). 10004: Classificação dos resíduos sólidos. Rio de Janeiro: Associação Brasileira de Normas Técnicas.

Odum, E. P. (1998). Ecología: el puente entre ciencia y sociedad (No. Sirsi) i9789701019061). McGraw-Hill Interamericana.

Pozzetti, V. C., \& Caldas, J. N. (2019). O descarte de resíduos sólidos no âmago da sustentabilidade. Revista de Direito Econômico e Socioambiental, 10(1), $183-205$.

Pozzetti, V. C., \& Caldas, J. N. (2019). O descarte de resíduos sólidos no âmago da sustentabilidade. Revista de Direito Econômico e Socioambiental, 10(1), $183-205$.

Programa de Pesquisa em Saneamento Básico, \& Bidone, F. R. A. (1999). Metodologias e técnicas de minimização, reciclagem, e reutilização de resíduos sólidos urbanos. ABES.

Santos, M. H. S., \& Marchesini, M. M. P. (2018). Logística reversa para a destinação ambientalmente sustentável dos resíduos de construção e demolição (RCD). Revista Metropolitana de Sustentabilidade, 8(2), 67-85.

Taglieber, J. E., \& Guerra, A. F. S. (2002). A dimensão ambiental na educação e as representações docentes. Encontro Perspectivas do Ensino de Biologia. São Paulo. Coletânea... VIII Encontro Perspectivas do Ensino de Biologia. São Paulo: FEUSP/EDUSP.

Veras, T. S., \& de Faria, V. A. (2019). Gestão De Resíduos Da Construção Civil-Investigação Sobre Políticas De Uso E Destinação Dos Resíduos Da Construção E Demolição Em Palmas-TO. Revista Integralização Universitária, (21), 131-144.

Wendland, E., \& Schalch, V. (2003). Pesquisas em meio ambiente: subsídios para a gestão de políticas públicas. RiMa. 